\title{
ICAM-1 Upregulation in Ethanol-Induced Fatty Murine Livers Promotes Injury and Sinusoidal Leukocyte Adherence after Transplantation
}

\author{
Tom P. Theruvath, ${ }^{1,2}$ Venkat K. Ramshesh, ${ }^{1}$ Zhi Zhong, ${ }^{1}$ Robert T. Currin, ${ }^{3}$ \\ Thomas Karrasch, ${ }^{4}$ and John J. Lemasters ${ }^{1,5}$ \\ ${ }^{1}$ Center for Cell Death, Injury \& Regeneration, Department of Pharmaceutical \& Biomedical Sciences, Medical University of \\ South Carolina, 280 Calhoun Street, MSC 140, Charleston, SC 29425, USA \\ ${ }^{2}$ Department of Surgery, Medical University of South Carolina, 280 Calhoun Street, MSC 140, Charleston, SC 29425, USA \\ ${ }^{3}$ Department of Cell \& Developmental Biology, University of North Carolina, Chapel Hill, NC 27599, USA \\ ${ }^{4}$ Department of Medicine, University of Regensburg, 93053 Regensburg, Germany \\ ${ }^{5}$ Department of Biochemistry \& Molecular Biology, Medical University of South Carolina, 280 Calhoun Street, \\ MSC 140, Charleston, SC 29425, USA
}

Correspondence should be addressed to John J. Lemasters, jjlemasters@musc.edu

Received 30 January 2012; Accepted 30 April 2012

Academic Editor: Peter Schemmer

Copyright (c) 2012 Tom P. Theruvath et al. This is an open access article distributed under the Creative Commons Attribution License, which permits unrestricted use, distribution, and reproduction in any medium, provided the original work is properly cited.

\begin{abstract}
Background. Transplantation of ethanol-induced steatotic livers causes increased graft injury. We hypothesized that upregulation of hepatic ICAM-1 after ethanol produces increased leukocyte adherence, resulting in increased generation of reactive oxygen species (ROS) and injury after liver transplantation (LT). Methods. C57BL/6 wildtype (WT) and ICAM-1 knockout (KO) mice were gavaged with ethanol $(6 \mathrm{~g} / \mathrm{kg}$ ) or water. LT was then performed into WT recipients. Necrosis and apoptosis, 4-hydroxynonenal (4HNE) immunostaining, and sinusoidal leukocyte movement by intravital microscopy were assessed. Results. Ethanol gavage of WT mice increased hepatic triglycerides 10 -fold compared to water treatment $(P<0.05)$. ICAM-1 also increased, but ALT was normal. At $8 \mathrm{~h}$ after LT of WT grafts, ALT increased 2-fold more with ethanol than water treatment $(P<0.05)$. Compared to ethanoltreated WT grafts, ALT from ethanol-treated KO grafts was 78\% less $(P<0.05)$. Apoptosis also decreased by $75 \%(P<0.05)$, and 4-HNE staining after LT was also decreased in ethanol-treated KO grafts compared to WT. Intravital microscopy demonstrated a 2 -fold decrease in leukocyte adhesion in KO grafts compared to WT grafts. Conclusions. Increased ICAM-1 expression in ethanoltreated fatty livers predisposes to leukocyte adherence after LT, which leads to a disturbed microcirculation, oxidative stress and graft injury.
\end{abstract}

\section{Introduction}

After cold ischemic liver storage for transplantation, reperfusion injury may lead to poor initial graft function and even graft failure. This injury is more severe and causes increased morbidity and mortality when steatotic donor livers are used $[1,2]$. Because of the increasing incidence of nonalcoholic steatohepatitis in the general population and the association of vehicular accidents with steatosis-causing alcohol use and abuse, an important fraction of potential human donor livers is steatotic. Such marginal steatotic livers are increasingly used as liver grafts because of the liver donor shortage and the expanding waiting list for liver transplantation.

Sinusoidal endothelial cells and hepatocytes are particularly susceptible to ischemia/reperfusion (I/R) injury and consequent apoptotic and necrotic cell death, as shown by both in vitro and in vivo studies [3-6]. After liver $\mathrm{I} / \mathrm{R}$, recruitment of neutrophils and other inflammatory cells aggravates injury $[7,8]$. Neutrophil recruitment also contributes to liver injury after endotoxin, sepsis, and chronic ethanol treatment [9-12]. Hepatic infiltration with neutrophils results in production of reactive oxygen species 
(ROS) and oxidative stress, resulting in neutrophil-mediated liver cell killing.

Intercellular adhesion molecule-1 (ICAM-1) is an endothelial- and leukocyte-associated transmembrane protein important in adherence of neutrophils to liver cells, including hepatocytes [13], and promotion of adherencedependent oxidant stress, a major factor in neutrophilmediated hepatocyte killing $[10,14]$. However in a previous study, antibody blockade of ICAM-1, although decreasing white blood cell adherence, did not protect against I/R injury in a rat model of lean liver transplantation [15]. The importance of ICAM-1 in fatty liver transplantation has not been assessed. Accordingly, we compared liver injury in a murine model of fatty liver transplantation using wildtype and ICAM-deficient liver grafts. Our results show that ethanol treatment increases hepatic ICAM-1. Such ICAM1 upregulation predisposes to leukocyte adherence, microcirculatory disturbances, oxidative stress, and increased graft injury after liver transplantation.

\section{Materials and Methods}

2.1. Ethanol Treatment and Donor Operation. All experiments were conducted using protocols approved by the Institutional Animal Care and Use Committee. Male C57BL/6 (wild-type) and ICAM-1-deficient mice, B6.129S4$I C A M 1^{t m 1 / c g r} / J$ on a C57BL/6 background, were gavaged with $6 \mathrm{~g} / \mathrm{kg}$ ethanol or water alone. Twelve hours after gavage, livers were harvested under ether anesthesia and stored in ice cold University of Wisconsin (UW), as previously described [16]. Time for the donor operation averaged $22 \mathrm{~min}$.

2.2. Recipient Operation. Livers from wild-type and ICAM1-deficient mice were transplanted without rearterialization into wild-type mouse recipients under ether anesthesia, as previously described [16]. Both donor and recipient mice weighed $19-24 \mathrm{~g}$. The recipient operation averaged $45 \mathrm{~min}$, and portal vein clamp time averaged $15 \mathrm{~min}$. For sham operations under ether anesthesia, wild-type and ICAM1-deficient mice were laparotomized. After $45 \mathrm{~min}$, the abdomen was closed.

2.3. Tissue Triglyceride Content. Liver tissues $(50 \mathrm{mg}$ ) of both wild type and ICAM-1 deficient mice were homogenized in water, and lipids were extracted into $\mathrm{CHCl}_{3}$ [17], dried in a vacuum centrifugal evaporator (Jouan RC 10.10, Thermo Scientific Inc., Atlanta, GA), and resuspended in $1 \mathrm{~mL}$ of $\mathrm{CHCl}_{3}$. An aliquot $(50 \mu \mathrm{L})$ was dried and resuspended in $100 \mu \mathrm{L}$ of isopropyl alcohol, $1 \%$ Triton X-100. Triacylglycerol (TAG) content was then determined using an enzymatic colorimetric method (Triglyceride Test Kit, Stanbio Laboratory, Boerne, TX).

2.4. Alanine Aminotransferase (ALT). Blood samples to measure ALT were collected from the inferior vena cava $8 \mathrm{~h}$ after transplantation for analysis by standard methods.
2.5. Histology. Histology was evaluated $8 \mathrm{~h}$ after liver transplantation. Liver tissues were fixed by immersion in $4 \%$ paraformaldehyde in phosphate-buffered saline and embedded in paraffin. Sections $(4 \mu \mathrm{m})$ were stained with hematoxylin and eosin (H\&E). Ten random fields were assessed for necrosis by standard morphologic criteria (e.g., loss of architecture, vacuolization, karyolysis, increased eosinophilia). Images were captured on a microscope (Zeiss Axiovert 100 microscope, Thornwood, NY), and the area percentage of necrosis was quantified using a computer program (AxioQuant, BD Bioimaging Systems, San Jose, CA).

2.6. Cell Death Immunohistochemistry. Terminal deoxynucleotidyl transferase-mediated dUTP nick-end labeling (TUNEL) was performed on paraffin sections using an in situ cell death detection kit (Roche Diagnostics, Penzberg, Germany). TUNEL-positive parenchymal and nonparenchymal cells were counted by light microscopy in 10 random highpower fields (HPFs).

2.7. Lipid Peroxidation Immunohistochemistry. Lipid peroxidation was assessed immunocytochemically by detecting 4-hydroxy-2-nonenal (HNE) adducts with a rabbit 4-HNE antibody (Alpha Diagnostic International, San Antonio, TX) with visualization by anti-rabbit IgG horse radish peroxidase (HRP) and diaminobenzidine (DAB) chromogen according to the manufacturer's instructions (DAKO corporation, Carpinteria, CA). The slides were then counterstained with hematoxylin.

2.8. Intravital Imaging of White Blood Cell Adherence. At $4 \mathrm{~h}$ after transplantation, recipients were anesthetized with pentobarbital $(50 \mathrm{mg} / \mathrm{kg})$ and connected to a small animal ventilator via a tracheostomy and respiratory tube (20-gauge catheter), as previously described [16]. Briefly, a catheter (0.4 mm inner diameter, Zeus, Inc., Orangeburg, SC) was inserted into the right carotid artery. Using a syringe pump, rhodamine $6 \mathrm{G}(1 \mu \mathrm{mol} /$ mouse $)$ was infused via the catheter over $20 \mathrm{~min}$. During this time a laparotomy was performed using the previous incision line. After prone positioning of the mouse, the liver was gently withdrawn from the abdominal cavity and placed over a glass coverslip on the stage of a Zeiss Axiovert 100 microscope (Thornwood, NY). Images of rhodamine $6 \mathrm{G}$ fluorescence were collected with a 40X 1.2 NA water-immersion objective lens through a spinning disk confocal imaging attachment (Attofluor CARV Optical Module, BD Bioimaging Systems, San Jose, CA) to a 12-bit cooled CCD camera (Hamamatsu, Bridgewater, NJ). In $10 \mathrm{sec}$ movies of 5 random fields per liver, white blood cells were scored for sticking (permanent adherence) and rolling (margination and slowing of white blood cell flow). Image analysis was performed in a blinded fashion using MetaFluor v.5.0 (Universal Imaging Corp., Downingtown, PA).

2.9. Statistical Analysis. Data are presented as means \pm S.E., unless otherwise noted. Statistical analysis was performed by 


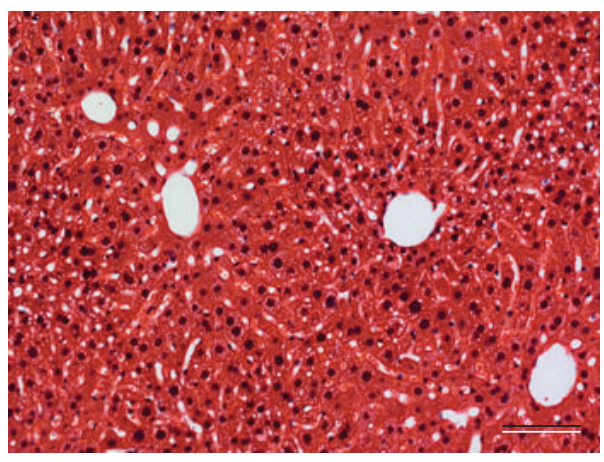

(a)

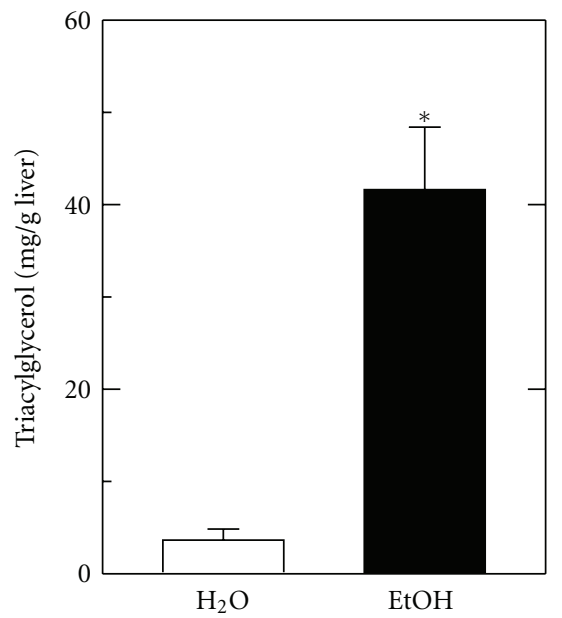

(c)

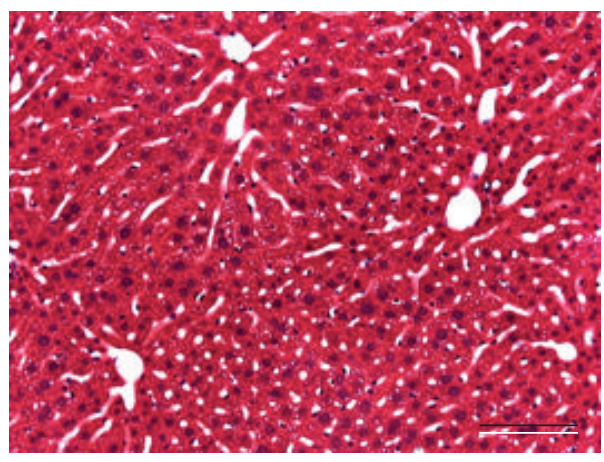

(b)

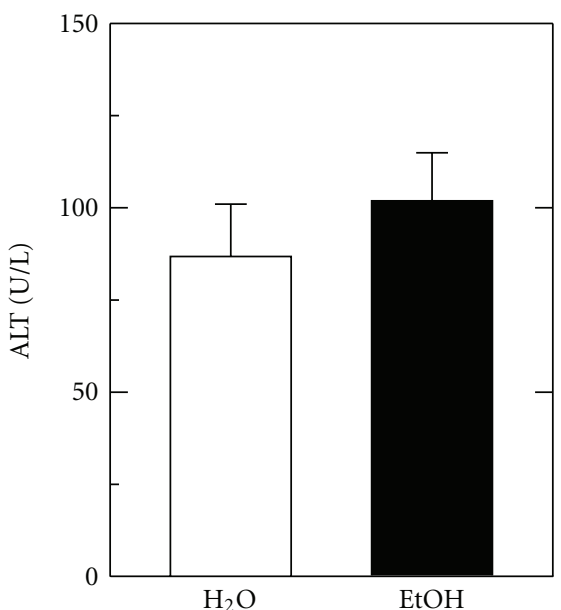

(d)

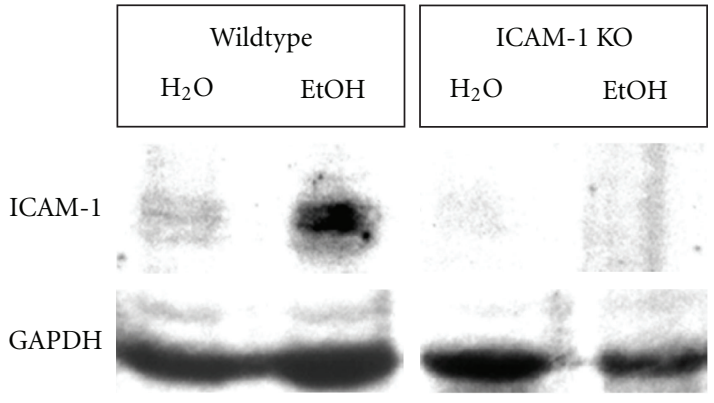

(e)

FIGURE 1: After ethanol gavage, mouse livers have increased fat content and ICAM-1 protein expression. Representative images of hepatic histology after ethanol gavage in wild type (a) and ICAM-1 deficient mice (b) are shown. Hepatic triacylglycerol (c), serum ALT (d), and hepatic ICAM-1 protein expression by Western blot (e) were assessed $12 \mathrm{~h}$ after water $\left(\mathrm{H}_{2} \mathrm{O}\right)$ and ethanol $(\mathrm{EtOH})$ gavage, as described in Section 2. (e) shows upregulation of ICAM-1 after ethanol treatment and the absence of ICAM-1 in ICAM-1 deficient (KO) mice. Size of individual groups was $3-4$. Bar is $50 \mu \mathrm{m} .{ }^{*} \mathrm{P}<0.05$ versus $\mathrm{H}_{2} \mathrm{O}$.

Student's $t$-test or ANOVA plus Student-Newman-Keuls test as appropriate, using $P<0.05$ as the criterion of significance.

\section{Results}

3.1. ICAM-1 Upregulation in Ethanol-Induced Fatty Livers. Wild type and ICAM-1 deficient mice were gavaged with ethanol or water, as described in Section 2. At $12 \mathrm{~h}$ after ethanol gavage, marked steatosis occurred to an equal extent in the livers of wild type and ICAM-1 deficient mice, which were indistinguishable histologically (compare Figures 1(a) and 1(b)). Overall, no differences in histology in livers of wild type and ICAM-1 deficient mice were observed either before or after ethanol treatment. After $12 \mathrm{~h}$, serum ALT levels were normal and comparable in both wild type and ICAM-1 deficient mice (Figure 1(c)). However, triacylglycerol levels increased 10-fold after ethanol treatment compared to water 


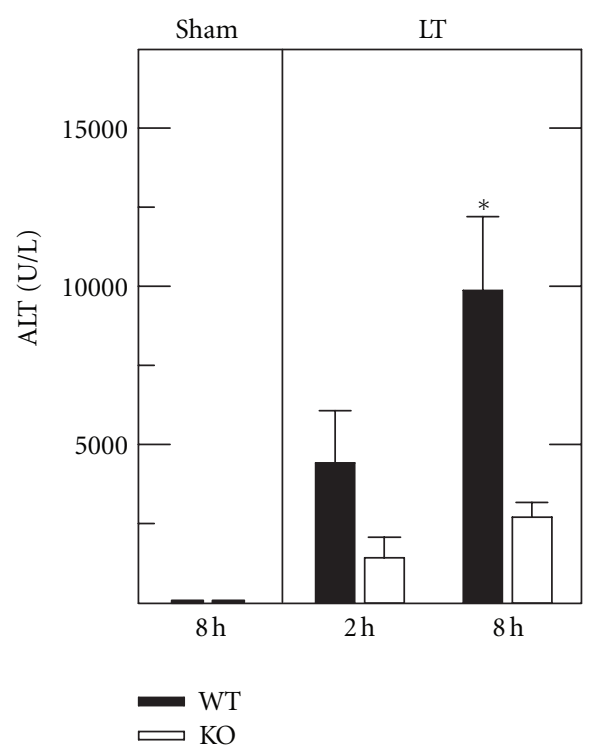

FIgURE 2: ALT release is decreased after transplantation of ICAM1 deficient fatty livers. Serum ALT was assessed $8 \mathrm{~h}$ after sham operation (Sham) and 2 and $8 \mathrm{~h}$ after mouse liver transplantation (LT), as described in Section 2. Transplants were performed into WT recipients after ethanol treatment of wild type (WT) or ICAM1 deficient (KO) liver donors. Sham groups had normal and comparable values to unoperated animals, and only sham is shown. Group sizes were sham WT and KO, 3; LT WT, 5; LT KO, 6. ${ }^{*} P<$ 0.05 versus $\mathrm{KO}$ grafts.

treatment $(P<0.01$, Figure $1(\mathrm{~d}))$. Western blotting of homogenized liver tissue revealed increased ICAM-1 expression at $12 \mathrm{~h}$ after ethanol treatment and, as expected, absent ICAM-1 expression in ICAM-1 deficient livers (Figure 1(e)). Overall, although ICAM-1 upregulation did not occur in ICAM-1 deficient mice, hepatic histology, steatosis, and ALT release were comparable in wild type and ICAM-1 deficient mice after acute ethanol treatment.

\subsection{Decreased ALT Release and Graft Necrosis after Trans-} plantation of ICAM-1 Deficient Fatty Livers. At $8 \mathrm{~h}$ after sham operation in ethanol-treated animals, wild type and ICAM-1 deficient mice had normal and comparable serum ALT averaging $118 \pm 13 \mathrm{U} / \mathrm{L}$ (Figure 2). In contrast, ALT increased markedly in recipient mice after ethanol treatment, $12 \mathrm{~h}$ storage, and transplantation of livers. At $2 \mathrm{~h}$ after transplantation, ALT increased to $4,431 \pm 1,636 \mathrm{U} / \mathrm{L}$ and $1,423 \pm 656 \mathrm{U} / \mathrm{L}(P=0.1)$, respectively, in recipients of wild type and ICAM-1 deficient livers. At $8 \mathrm{~h}$ after transplantation, ALT increased to $9,870 \pm 2,344 \mathrm{U} / \mathrm{L}$ and $2709 \pm 458 \mathrm{U} / \mathrm{L}$, respectively $(P<0.05)$ (Figure 2$)$. Thus, at $8 \mathrm{~h}$ following transplantation, ALT was $72 \%$ less in ICAM knockout than wild type liver recipients.

Graft injury was also assessed histologically. Liver histology was normal and indistinguishable in wild type and ICAM-1 deficient mice with and without sham operation (Figure 3(a) and data not shown). At $8 \mathrm{~h}$ after transplantation of wild type livers, large areas of necrosis were present with a predominantly pericentral and midzonal distribution (Figure 3(b)). By comparison, necrosis was decreased after transplantation of ICAM-1 deficient livers (Figure 3(c)). Morphometry revealed a decrease of hepatic necrosis from $25 \pm 5.3 \%$ after wild type liver transplantation to $6.5 \pm 3.1 \%$ after transplantation of ICAM-1 deficient livers $(P<0.05)$ (Figure 3(d)). Thus, transplantation of ICAM-1 deficient fatty livers decreased hepatic necrosis by three quarters.

3.3. Decreased Graft Apoptosis after Transplantation of ICAM1 Deficient Fatty Livers. TUNEL was performed on tissue sections to assess double-stranded DNA breaks that are characteristic of apoptosis. TUNEL-positive cells were rare in wild type and ICAM-1 livers with and without sham operation, averaging less than 1 cell/HPF (Figures $4(\mathrm{a})$ and $4(\mathrm{~d})$, and data not shown). At $8 \mathrm{~h}$ after transplantation with wild type livers, TUNEL in nonnecrotic areas increased to $12.2 \pm 4.8$ cells/HPF without apparent zonal localization (Figures 4(b) and 4(d)). After transplantation of ICAM1 deficient livers, TUNEL decreased by about two-thirds to $3.5 \pm 1.1$ cells/HPF $(P<0.05$, Figures $4(\mathrm{c})$ and $4(\mathrm{~d}))$. As a percentage of all cells, TUNEL was $2.6 \pm 1.3 \%$ after transplantation of wild type livers versus $0.7 \pm 0.3 \%$ after transplantation of ICAM-1 deficient livers $(P<0.05)$.

3.4. Decreased White Blood Cell Adhesion in Fatty ICAM-1 Deficient Liver Grafts. At $4 \mathrm{~h}$ after sham operation, intravital confocal microscopy revealed bright fluorescence of rhodamine 6G-labeled white blood cells moving through hepatic sinusoids. No differences were seen in wild type or ICAM1 deficient mice after sham operation and only occasional margination (rolling) and sticking of rhodamine 6G-labeled cells were noted in sinusoids (Figures 5(a) and 5(b), and Video A of supplemental data in Supplementary Material available online at doi:10.11/2012/480893). By contrast, at $4 \mathrm{~h}$ after liver transplantation of fatty wild type livers, marginating (rolling) and adherent (stickers) rhodamine 6G-stained cells increased markedly (Figure 5, Video B of supplemental data).

After transplantation of ICAM-1 deficient livers, fewer nonmobile rhodamine 6G-stained cells (stickers) were present in hepatic sinusoids (Video C of supplemental data). Rhodamine 6G-stained rollers and stickers were scored and counted for each liver. In ethanol-treated sham-operated livers, $1.6 \pm 0.36$ stickers $/ 100 \mu \mathrm{m}^{2}$ were identified. After transplantation of fatty WT livers, stickers increased to $15.0 \pm$ $4.04 / 100 \mu \mathrm{m}^{2}$, a more than 8 -fold increase. Similarly, rollers increased by 2.5 -fold after wild type transplantation compared to sham (Figures 5(a) and 5(b)). After transplantation of ICAM-1 deficient livers, stickers decreased 54\% to $6.9 \pm$ 1.04 per $100 \mu \mathrm{m}^{2}(P<0.05$ versus wild type) (Figure 5(b)). By contrast, rollers did not decrease in ICAM-1 compared to wild type liver grafts (Figure 5(a)). Thus, sinusoidal adherence (sticking) but not initial margination (rolling) of white blood cells was decreased in ICAM-1 deficient compared to wild type grafts after liver transplantation. 


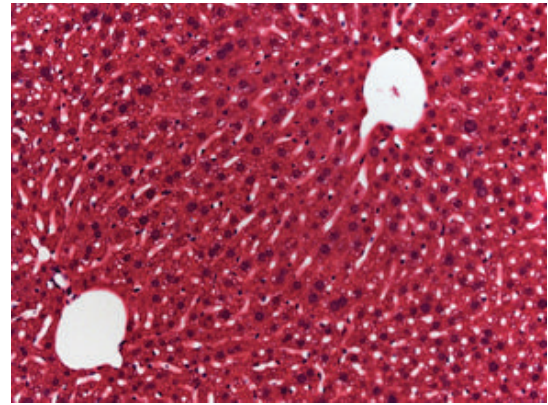

(a)

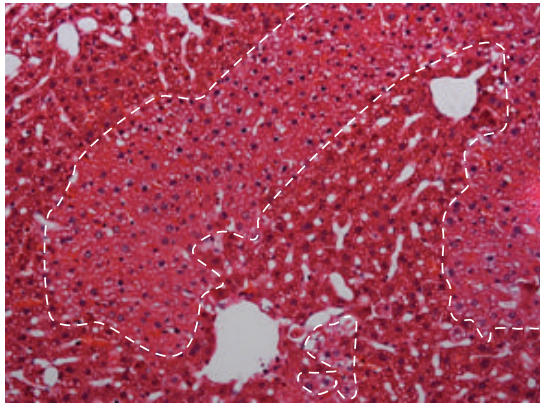

(b)

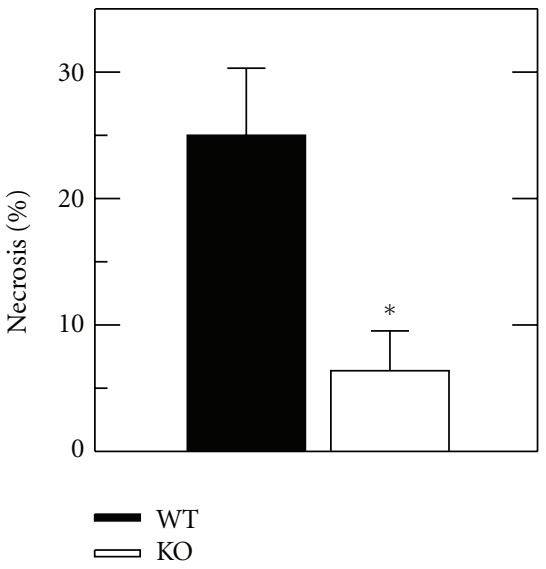

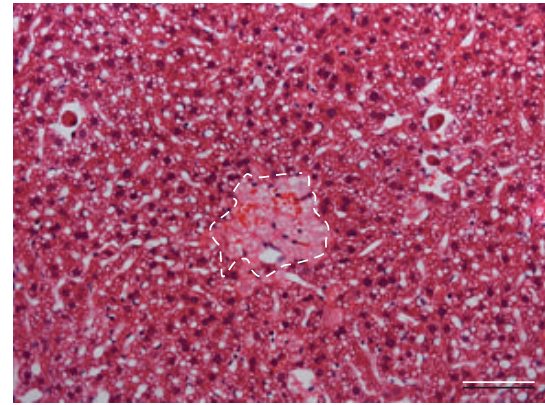

(c)

(d)

FIgURE 3: Necrosis is decreased after transplantation of ICAM-1 deficient fatty livers. Mouse livers were transplanted, as described in Section 2. At $8 \mathrm{~h}$ postoperatively, necrosis was assessed by H\&E histology after sham operation (a), transplantation of wild type (WT) livers (b), and transplantation of ICAM-1 deficient (KO) livers (c). (d) shows necrosis as percent area in liver sections averaged from 5 livers per group. Necrosis in sham-operated WT and KO livers was absent. Bar is $50 \mu \mathrm{m} .{ }^{*} P<0.05$ versus WT grafts.

3.5. Decreased Oxidative Stress after Transplantation of Fatty ICAM-1 Deficient Livers. We used 4-HNE immunocytochemistry as a marker of oxidative stress in liver grafts at $2 \mathrm{~h}$ and $8 \mathrm{~h}$ after reperfusion. HNE adduct formation is a consequence of lipid peroxidation resulting from ROS generation. In livers of ethanol-treated wild type and ICAM1 deficient mice at $8 \mathrm{~h}$ after sham operation, HNE brown staining was nearly undetectable (Figures 6(a) and 6(b)). By contrast, at $2 \mathrm{~h}$ after transplantation of fatty wild type livers, HNE staining in the cytoplasm and nuclei of hepatocytes was present in a mosaic pattern throughout the tissue sections (Figure 6(c)). After $8 \mathrm{~h}$, HNE staining became confluent in midzonal and pericentral areas with sparing of periportal areas (Figure 6(e)).

Compared to wild type, far fewer hepatocytes of fatty ICAM- 1 deficient liver grafts stained for HNE both at $2 \mathrm{~h}$ and $8 \mathrm{~h}$ after reperfusion (Figures 6(d) and 6(f)). Thus, oxidative stress in ICAM-1 deficient fatty liver grafts was decreased compared to wild type fatty grafts.

\section{Discussion}

The limiting factor in clinical liver transplantation is donor shortage, which leads to death of patients on the waiting list.
Expanding the donor pool by including marginal steatotic donor livers would help shorten wait times and increase the availability of donor livers for transplantation. However, such increased use requires overcoming the increased susceptibility of fatty liver grafts to poor initial function and failure. Targeting specific pathways to decrease reperfusion injury of cold stored steatotic livers might thus be a beneficial approach to improve the function and survival of fatty liver grafts.

The aim of the present study was to evaluate the importance of ICAM-1 in graft injury after transplantation of ethanol-induced steatotic mouse livers. ICAM-1 has previously been shown to contribute to hepatic injury after various nonsurgical liver stresses $[18,19]$ and ICAM-1 blockade demonstrated less white blood cell adherence after lean rat liver transplantation [15]. We tested the hypothesis that ICAM-1 upregulation in ethanol-induced fatty livers leads to necrosis and apoptosis after transplantation through sinusoidal leukocyte adherence and subsequent ROS generation. We evaluated graft injury by enzyme release, necrosis and apoptosis, white blood cell adherence by intravital microscopy, and oxidative stress by HNE immunocytochemistry after transplantation. Our findings demonstrate that ethanol treatment upregulates ICAM-1 expression and that 


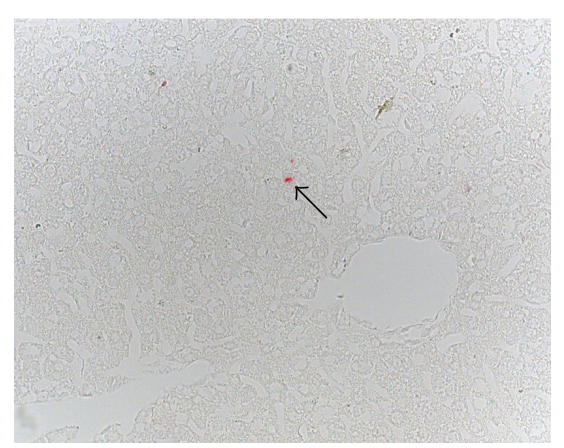

(a)

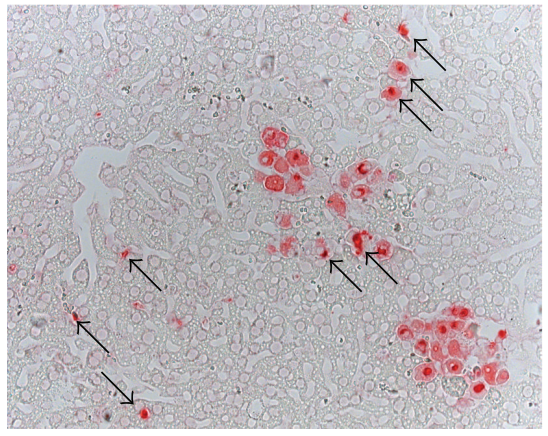

(b)

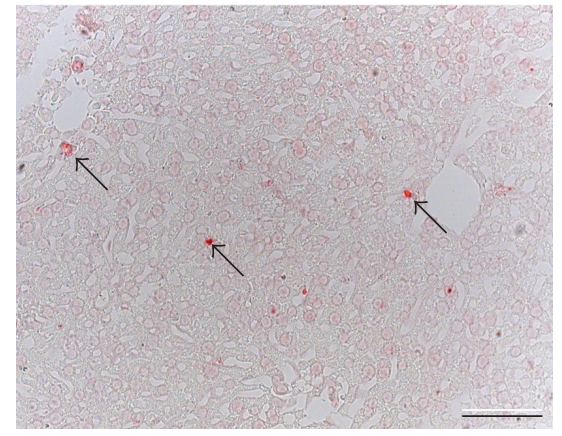

(c)

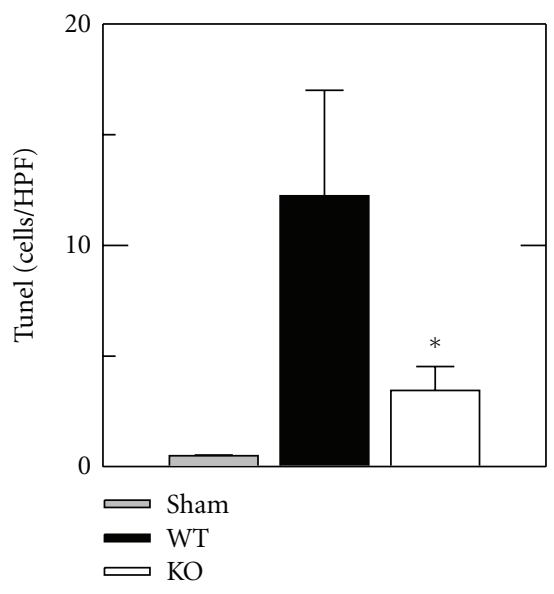

(d)

Figure 4: Apoptosis is decreased after transplantation of ICAM-1 deficient fatty livers. Mouse livers were transplanted, as described in Section 2. At $8 \mathrm{~h}$ postoperatively, TUNEL was assessed in tissue sections after sham operation (a), transplantation of wild type (WT) livers (b), and transplantation of ICAM-1 deficient (KO) livers (c). (d) quantifies the TUNEL-positive cells per high power field (HPF). TUNEL for WT sham was virtually zero and comparable to KO sham, and only $\mathrm{KO}$ sham is plotted. Bar is $50 \mu \mathrm{m}$. ${ }^{*} P<0.05$ versus WT grafts.

ICAM-1 deficiency decreases injury, leukocyte adherence and oxidative stress in ethanol-induced steatotic liver grafts. Our results are consistent with the conclusion that protection in ICAM-1 deficient grafts is the consequence of decreased sinusoidal adherence of white blood cells and decreased ROS formation.

Previous studies utilizing rodents demonstrate that doses of $5-6 \mathrm{~g} / \mathrm{kg}$ ethanol increase hepatic triglyceride and decrease graft survival after transplantation substantially $[20,21]$. Such a dose produces a peak blood ethanol concentration of about $370 \mathrm{mg} / \mathrm{dL}$ after $2 \mathrm{~h}$ in rats, which declines to undetectable levels in 8 to $10 \mathrm{~h}$. No respiratory suppression is observed in these ethanol-treated animals receiving this treatment. In humans, fatty liver occurs to a similar extent after an acute ethanol binge [22]. Accordingly, rodent models have been used for decades to investigate the extent of ethanol-induced liver steatosis in a variety of contexts. In our experimental setting in mice, a single high dose of ethanol $(6 \mathrm{~g} / \mathrm{kg})$ administered by gavage produced prominent hepatic steatosis $12 \mathrm{~h}$ later and a 10 -fold increase of hepatic triacylglycerol content (Figure 1). Steatosis was associated with increased hepatic ICAM-1 expression (Figure 1(e)). Although blood alcohol peaked at about $580 \mathrm{mg} / \mathrm{dL}$ at $40 \mathrm{~min}$ after this treatment (data not shown), increases of necrosis, apoptosis, and serum ALT were negligible, and mortality did not occur. Interestingly, ethanol-induced upregulation of ICAM-1 alone did not increase white blood cell adherence before cold storage and reperfusion (Figure 5 and Video A of supplemental data). However, after transplantation of ethanol-induced fatty livers, white blood cell adherence increased markedly, an effect attenuated by more than half in ICAM-1 deficient liver grafts (Figure 5 and Videos B and C of supplemental data). Injury was also decreased in ICAM-1 deficient grafts (Figures 2, 3, and 4).

Previously in a study of warm liver $\mathrm{I} / \mathrm{R}$, antibody against ICAM-1 did not improve outcome when used alone but only when combined with antibodies against lymphocytefunction-associated antigen-1 (LFA-1) and beta 2 integrin, (CD-18), which are binding partners for ICAM-1 present on leukocyte membrane surfaces [23]. Similarly, after lean liver transplantation, ICAM-1 antibodies failed to prevent graft injury, although leukocyte adherence was decreased [15]. Thus, ICAM-1-dependent leukocyte adherence may itself be insufficient to cause liver injury and instead may act synergistically with other alterations to cause tissue damage. In the setting of transplantation of ethanol-induced 


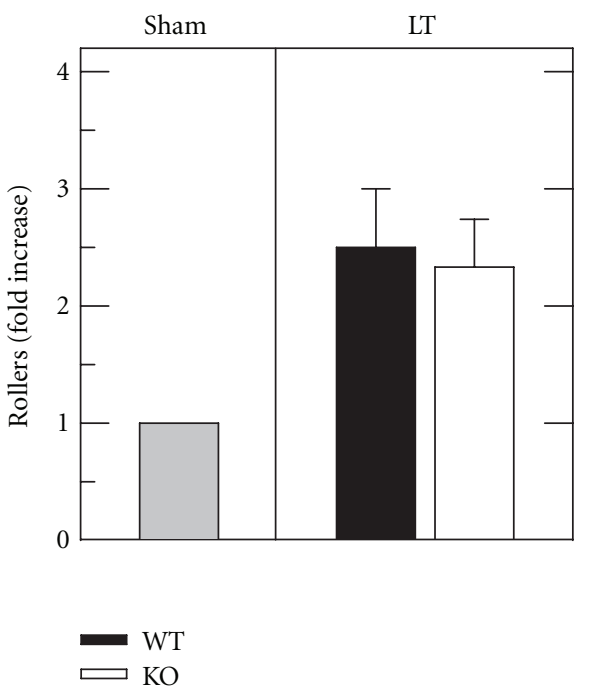

(a)

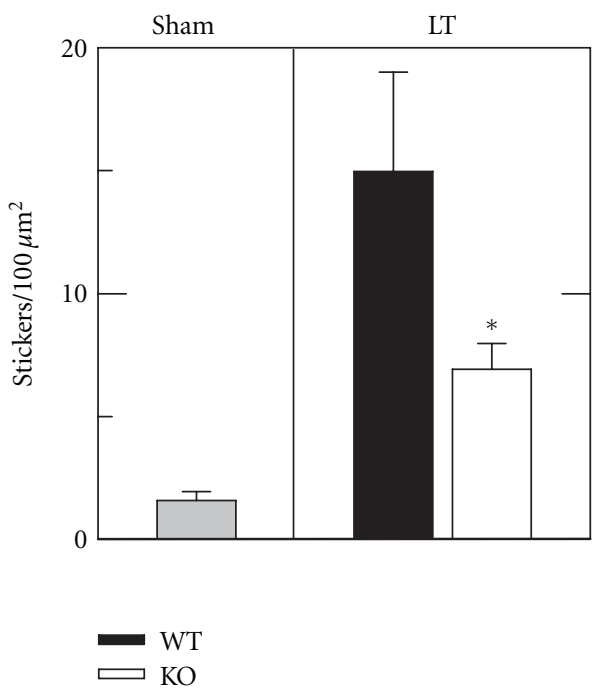

(b)

FIGURE 5: ICAM-1 deficient grafts show decreased leukocyte adherence but unchanged rolling margination after ethanol-induced fatty mouse liver transplantation. Livers were transplanted (LT) or subjected to sham operation (Sham) and visualized by intravital confocal microscopy of rhodamine $6 \mathrm{G}$ fluorescence after $4 \mathrm{~h}$, as described in Section 2. Rhodamine 6G-fluorescing leukocytes were scored for slow flow rolling margination (Rollers, (a)) and no flow adherence to sinusoidal walls (Stickers, (b)) as either fold increase versus sham (a) or as absolute number per $\mu \mathrm{m}^{2}$ (b). WT and KO sham groups were comparable, and only the KO sham group is plotted. Individual group size was 4. ${ }^{*} P<0.05$ versus WT grafts.

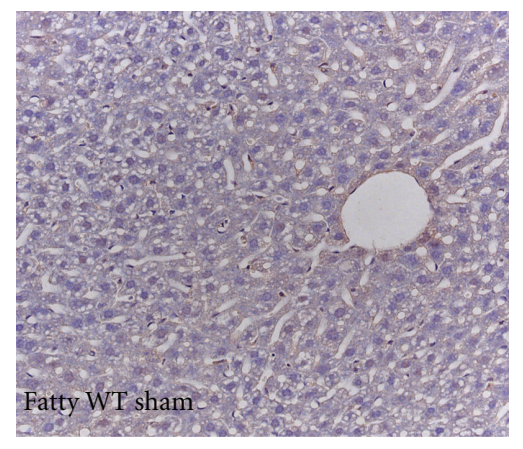

(a)

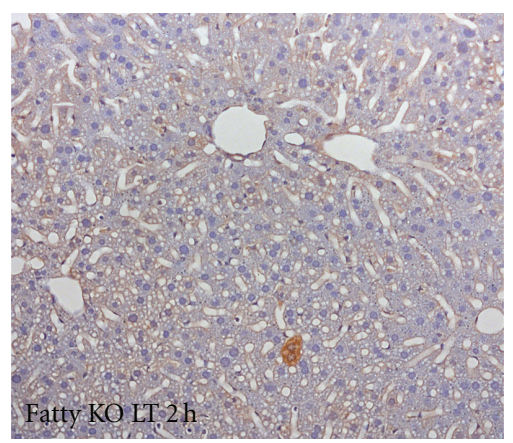

(d)

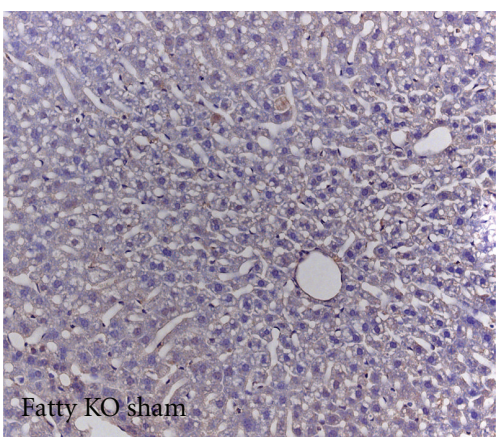

(b)

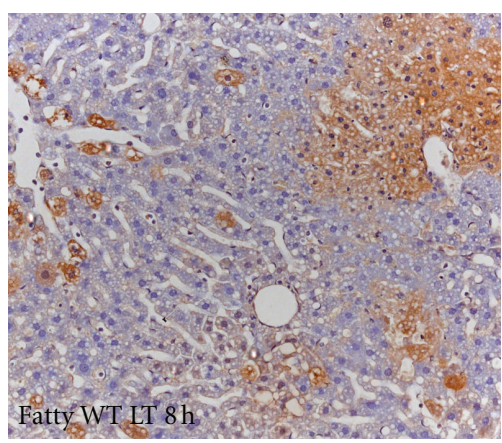

(e)

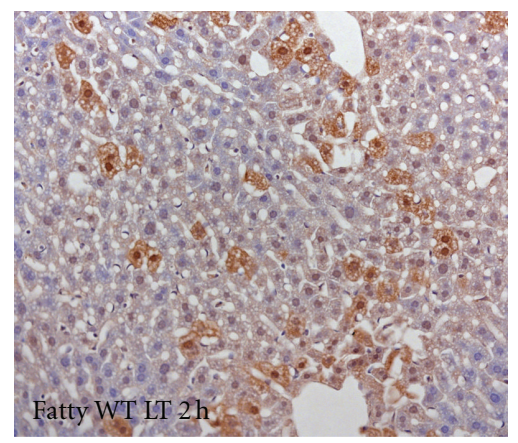

(c)

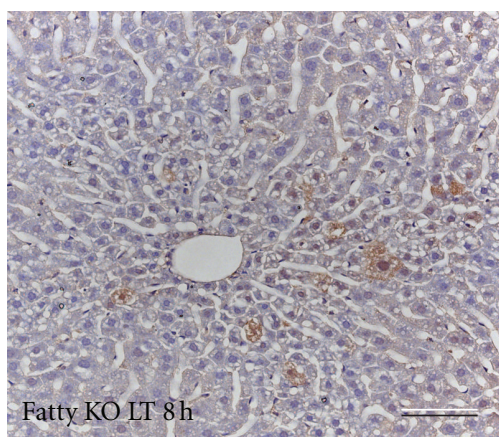

(f)

FIgURE 6: Decreased lipid peroxidation occurs in ICAM-1 deficient liver grafts. At 2 and $8 \mathrm{~h}$ after transplantation or sham operation, immunohistochemical staining was performed for 4-hydroxynonenal-modified proteins (4-HNE, brown), as described in Section 2. Panels are sham-operated wild type (WT) livers (a), sham-operated ICAM-1 deficient (KO) livers (b), WT liver grafts at $2 \mathrm{~h}$ after reperfusion (c), KO liver grafts at $2 \mathrm{~h}$ after reperfusion (d), WT liver grafts at $8 \mathrm{~h}$ after reperfusion (e), and KO liver grafts at $8 \mathrm{~h}$ after reperfusion (f). Brown HNE immunoreactivity was present in WT grafts after 2 and $8 \mathrm{~h}((\mathrm{c})$ and (e)), which was markedly decreased in KO livers ((d) and (f)). Size of groups was 4 . Bar is $50 \mu \mathrm{m}$. 
fatty livers, our studies would suggest that ethanol-induced sinusoidal ICAM-1 upregulation is a first hit that alone is insufficient to cause hepatic injury. After transplantation, however, activation of leukocytes may represent a second hit, which when combined with the first hit causes hepatic necrosis, apoptosis, and enzyme release. ICAM-1 in our setting of fatty liver transplantation had more impact on injury than in previous models of warm hepatic I/R and lean liver transplantation. Ethanol-induced upregulation of ICAM-1 prior to I/R or transplantation stress may be particularly important in predisposing livers to injury, and ICAM1 upregulation could potentially represent a biomarker to susceptibility of fatty livers to storage/reperfusion injury in human clinical transplantation.

Although leukocyte adherence (sticking) was decreased in ICAM-1 deficient liver grafts, rolling margination of leukocytes was unchanged (Figure 5, Videos B and C in supplemental data). These observations are consistent with earlier studies showing that endothelial selectins mediate rolling margination of leukocytes in response to chemokines and other proinflammatory signals, whereas ICAM-1 mediates adherence and infiltration into tissue [24].

ROS production by ischemic tissue after reperfusion is widely held as a major factor contributing to $\mathrm{I} / \mathrm{R}$ injury. ROS generation also contributes to cold storage/reperfusion injury [21, 25-32]. ROS induces tissue damage by activating mitochondrial pathways leading to necrosis and apoptosis and through direct attack on proteins, lipids, and DNA. HNE is a product of ROS-dependent peroxidation of $\omega-6$ polyunsaturated fatty acids, such as linoleic and arachidonic acid [33]. HNE reacts with protein sulfhydryls to form covalent adducts that can be detected by immunocytochemistry. In the present study, HNE adducts developed after wild type liver transplantation that were markedly decreased in ICAM-1 deficient liver grafts (Figure 6). Even at 2 hours after transplantation before onset of necrosis, HNE adducts were increased in wild type grafts but markedly decreased in ICAM-1 deficient grafts (Figure 6). These findings indicate that ROS production is temporally upstream of necrosis, which does not occur maximally until 4 or more hours after transplantation (Figures 2 and 3 and data not shown, see also $[25,26,34-36])$. Thus, ICAM-1-dependent leukocyte margination likely contributes to ROS generation and graft injury after wild type liver transplantation.

In summary, our results demonstrate involvement of ICAM-1 in storage/reperfusion injury to ethanol-induced fatty liver grafts. Prior to transplantation, ethanol treatment causes steatosis and upregulation of ICAM-1 expression in donor wild type livers. Such ICAM-1 upregulation was associated with increased leukocyte adherence, ROS generation, and injury to liver grafts. Thus, ICAM-1 upregulation and signaling in fatty liver grafts could represent a biomarker and target to identify and decrease susceptibility to storage/reperfusion injury of fatty liver grafts. However, the effects of chronic alcohol exposure or other means of fatty liver induction on graft injury, oxidative stress, and leukocyte recruitment may be different from those observed after acute ethanol administration. Thus, future studies will be needed to determine what benefit, if any, ICAM-1 targeting might have on fatty liver grafts in human clinical liver transplantation.

\section{Abbreviations}

$\begin{array}{ll}\text { ALT: } & \text { Alanine aminotransferase } \\ \text { ATP: } & \text { Adenosine triphosphate } \\ \text { CD-18: } & \text { Beta } 2 \text { integrin } \\ \text { DAB: } & 3,3^{\prime} \text {-diaminobenzidine } \\ \text { H\&E: } & \text { Hematoxylin and eosin } \\ \text { HNE: } & \text { 4-hydroxynonenal } \\ \text { HPF: } & \text { High power field } \\ \text { HRP: } & \text { Horse radish peroxidase } \\ \text { ICAM-1: } & \text { Intercellular adhesion molecule-1 } \\ \text { I/R: } & \text { Ischemia/reperfusion } \\ \text { LFA-1: } & \text { lymphocyte function-associated antigen-1 } \\ \text { LT: } & \text { Liver transplantation } \\ \text { ROS: } & \text { Reactive oxygen species } \\ \text { TUNEL: } & \text { Terminal deoxynucleotidyl transferase-mediated } \\ & \text { dUTP nick-end labeling } \\ \text { UW: } & \text { University of Wisconsin cold storage solution. }\end{array}$

\section{Acknowledgments}

The authors are grateful to Dr. Rosalind Coleman's laboratory at the University of North Carolina at Chapel Hill for providing advice and assistance in performing tissue triglyceride measurements. This work was supported, in part, by Grants DK37034, DK73336, DK70844, and C06 RR015455 from the National Institutes of Health and Grant DFG TH1328/1-1 from the Deutsche Forschungsgemeinschaft. Dr. T. Theruvath is the recipient of an American Liver Foundation Postdoctoral Research Fellowship Award. Portions of this work were presented at Experimental Biology 2007, Washington DC, April 28-May 2, 2007 [37], and American College of Surgeons Annual Clinical Congress 2009, Chicago, October 11-15, 2009 [38].

\section{References}

[1] W. A. Marsman, R. H. Wiesner, L. Rodriguez et al., "Use of fatty donor liver is associated with diminished early patient and graft survival," Transplantation, vol. 62, no. 9, pp. 12461251, 1996.

[2] L. McCormack, H. Petrowsky, W. Jochum, B. Mullhaupt, M. Weber, and P. A. Clavien, "Use of severely steatotic grafts in liver transplantation: a matched case-control study," Annals of Surgery, vol. 246, no. 6, pp. 940-946, 2007.

[3] J. C. Caldwell-Kenkel, R. T. Currin, Y. Tanaka, R. G. Thurman, and J. J. Lemasters, "Reperfusion injury to endothelial cells following cold ischemic storage of rat livers," Hepatology, vol. 10, no. 3, pp. 292-299, 1989.

[4] J. S. Kim, L. He, and J. J. Lemasters, "Mitochondrial permeability transition: a common pathway to necrosis and apoptosis," Biochemical and Biophysical Research Communications, vol. 304, no. 3, pp. 463-470, 2003.

[5] H. A. Rüdiger, R. Graf, and P. A. Clavien, "Liver ischemia: apoptosis as a central mechanism of injury," Journal of Investigative Surgery, vol. 16, no. 3, pp. 149-159, 2003. 
[6] Y. Takei, I. Marzi, W. S. Gao, G. J. Gores, J. J. Lemasters, and R. G. Thurman, "Leukocyte adhesion and cell death following orthotopic liver transplantation in the rat," Transplantation, vol. 51, no. 5, pp. 959-965, 1991.

[7] H. Jaeschke, A. Farhood, and C. W. Smith, "Neutrophils contribute to ischemia/reperfusion injury in rat liver in vivo," The FASEB Journal, vol. 4, no. 15, pp. 3355-3359, 1990.

[8] H. Jaeschke, "Mechanisms of reperfusion injury after warm ischemia of the liver," Journal of Hepato-Biliary-Pancreatic Surgery, vol. 5, no. 4, pp. 402-408, 1998.

[9] A. P. Bautista, "Chronic alcohol intoxication induces hepatic injury through enhanced macrophage inflammatory protein-2 production and intercellular adhesion molecule-1 expression in the liver," Hepatology, vol. 25, no. 2, pp. 335-342, 1997.

[10] J. S. Gujral, J. Liu, A. Farhood, J. A. Hinson, and H. Jaeschke, "Functional importance of ICAM-1 in the mechanism of neutrophil-induced liver injury in bile duct-ligated mice," American Journal of Physiology, vol. 286, no. 3, pp. G499G507, 2004.

[11] H. Jaeschke, A. Farhood, and C. W. Smith, "Neutrophilinduced liver cell injury in endotoxin shock is a CD11b/CD18dependent mechanism," American Journal of Physiology, vol. 261, no. 6, part 1, pp. G1051-G1056, 1991.

[12] R. G. Molnar, P. Wang, A. Ayala, P. E. Ganey, R. A. Roth, and I. H. Chaudry, "The role of neutrophils in producing hepatocellular dysfunction during the hyperdynamic stage of sepsis in rats," Journal of Surgical Research, vol. 73, no. 2, pp. 117-122, 1997.

[13] A. R. Nagendra, J. K. Mickelson, and C. W. Smith, "CD18 integrin and CD54-dependent neutrophil adhesion to cytokinestimulated human hepatocytes," American Journal of Physiology, vol. 272, no. 3, part 1, pp. G408-G416, 1997.

[14] H. Jaeschke, Y. S. Ho, M. A. Fisher, J. A. Lawson, and A. Farhood, "Glutathione peroxidase-deficient mice are more susceptible to neutrophil-mediated hepatic parenchymal cell injury during endotoxemia: importance of an intracellular oxidant stress," Hepatology, vol. 29, no. 2, pp. 443-450, 1999.

[15] M. Rentsch, S. Post, P. Palma, G. Lang, M. D. Menger, and K. Messmer, "Anti-ICAM-1 blockade reduces postsinusoidal WBC adherence following cold ischemia and reperfusion, but does not improve early graft function in rat liver transplantation," Journal of Hepatology, vol. 32, no. 5, pp. 821828, 2000.

[16] T. P. Theruvath, Z. Zhong, R. T. Currin, V. K. Ramshesh, and J. J. Lemasters, "Endothelial nitric oxide synthase protects transplanted mouse livers against storage/reperfusion injury: role of vasodilatory and innate immunity pathways," Transplantation Proceedings, vol. 38, no. 10, pp. 3351-3357, 2006.

[17] E. G. Bligh and W. J. Dyer, "A rapid method of total lipid extraction and purification," Canadian Journal of Biochemistry and Physiology, vol. 37, no. 8, pp. 911-917, 1959.

[18] B. K. Gunawan, Z. X. Liu, D. Han, N. Hanawa, W. A. Gaarde, and N. Kaplowitz, "c-Jun N-terminal kinase plays a major role in murine acetaminophen hepatotoxicity," Gastroenterology, vol. 131, no. 1, pp. 165-178, 2006.

[19] Y. Wang, R. Singh, J. H. Lefkowitch, R. M. Rigoli, and M. J. Czaja, "Tumor necrosis factor-induced toxic liver injury results from JNK2-dependent activation of caspase- 8 and the mitochondrial death pathway," The Journal of Biological Chemistry, vol. 281, no. 22, pp. 15258-15267, 2006.

[20] R. Ylikahri, "Metabolic effects of alcohol," Duodecim, vol. 88, no. 3, pp. 247-257, 1972.
[21] Z. Zhong, H. Connor, R. P. Mason et al., "Destruction of Kupffer cells increases survival and reduces graft injury after transplantation of fatty livers from ethanol-treated rats," Liver Transplantation and Surgery, vol. 2, no. 5, pp. 383-387, 1996.

[22] R. Scheig, "Effects of ethanol on the liver," American Journal of Clinical Nutrition, vol. 23, no. 4, pp. 467-473, 1970.

[23] S. Marubayashi, Y. Oshiro, T. Maeda et al., "Protective effect of monoclonal antibodies to adhesion molecules on rat liver ischemia-reperfusion injury," Surgery, vol. 122, no. 1, pp. 4552, 1997.

[24] K. E. Caputo and D. A. Hammer, "Adhesive dynamics simulation of G-protein-mediated chemokine-activated neutrophil adhesion," Biophysical Journal, vol. 96, no. 8, pp. 2989-3004, 2009.

[25] H. Jaeschke and J. J. Lemasters, "Apoptosis versus oncotic necrosis in hepatic ischemia/reperfusion injury," Gastroenterology, vol. 125, no. 4, pp. 1246-1257, 2003.

[26] J. J. Lemasters and R. G. Thurman, "Reperfusion injury after liver preservation for transplantation," Annual Review of Pharmacology and Toxicology, vol. 37, pp. 327-338, 1997.

[27] H. Jaeschke, C. V. Smith, and J. R. Mitchell, "Hypoxic damage generates reactive oxygen species in isolated perfused rat liver," Biochemical and Biophysical Research Communications, vol. 150, no. 2, pp. 568-574, 1988.

[28] M. Ozaki, S. S. Deshpande, P. Angkeow et al., "Inhibition of the Racl GTPase protects against nonlethal ischemia/reperfusion-induced necrosis and apoptosis in vivo," The FASEB Journal, vol. 14, no. 2, pp. 418-429, 2000.

[29] T. G. Lehmann, M. D. Wheeler, R. F. Schwabe et al., "Gene delivery of $\mathrm{Cu} / \mathrm{Zn}$-superoxide dismutase improves graft function after transplantation of fatty livers in the rat," Hepatology, vol. 32, no. 6, pp. 1255-1264, 2000.

[30] T. G. Lehmann, M. D. Wheeler, R. Schoonhoven, H. Bunzendahl, R. J. Samulski, and R. G. Thurman, "Delivery of Cu/Znsuperoxide dismutase genes with a viral vector minimizes liver injury and improves survival after liver transplantation in the rat," Transplantation, vol. 69, no. 6, pp. 1051-1057, 2000.

[31] T. G. Lehmann, M. D. Wheeler, M. Froh et al., "Effects of three superoxide dismutase genes delivered with an adenovirus on graft function after transplantation of fatty livers in the rat," Transplantation, vol. 76, no. 1, pp. 28-37, 2003.

[32] Z. Zhong and J. J. Lemasters, "Role of free radicals in failure of fatty liver grafts caused by ethanol," Alcohol, vol. 34, no. 1, pp. 49-58, 2004.

[33] W. G. Siems, T. Grune, B. Beierl, H. Zollner, and H. Esterbauer, "The metabolism of 4-hydroxynonenal, a lipid peroxidation product, is dependent on tumor age in Ehrlich mouse ascites cells," EXS, vol. 62, pp. 124-135, 1992.

[34] J. J. Lemasters, "Dying a thousand deaths: redundant pathways from different organelles to apoptosis and necrosis," Gastroenterology, vol. 129, no. 1, pp. 351-360, 2005.

[35] T. P. Theruvath, Z. Zhong, P. Pediaditakis et al., "Minocycline and N-methyl-4-isoleucine cyclosporin (NIM811) mitigate storage/reperfusion injury after rat liver transplantation through suppression of the mitochondrial permeability transition," Hepatology, vol. 47, no. 1, pp. 236-246, 2008.

[36] Z. Zhong, G. E. Arteel, H. D. Connor et al., "Binge drinking disturbs hepatic microcirculation after transplantation: prevention with free radical scavengers," Journal of Pharmacology and Experimental Therapeutics, vol. 290, no. 2, pp. 611-620, 1999. 
[37] T. P. Theruvath, Z. Zhong, V. K. Ramshesh, R. T. Currin, T. Karrasch, and J. J. Lemasters, "ICAM-1 upregulation in fatty livers of ethanol-treated donor mice promotes injury and sinusoidal leukocyte adherence after transplantation," The FASEB Journal, vol. 21, article A1218, 2007.

[38] T. P. Theruvath, K. D. Chavin, Z. Zhong, and J. J. Lemasters, "ICAM-1 upregulation in alcohol-induced fatty mouse livers promotes reperfusion injury after tramsplantation through increased oxidative stress and downstream leukocyte adherence," Journal of the American College of Surgeons, vol. 209, no. 3, supplement, p. S60, 2009. 


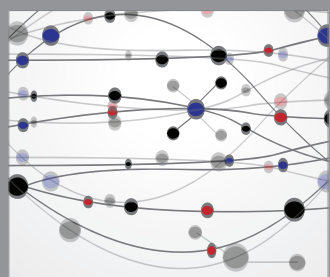

The Scientific World Journal
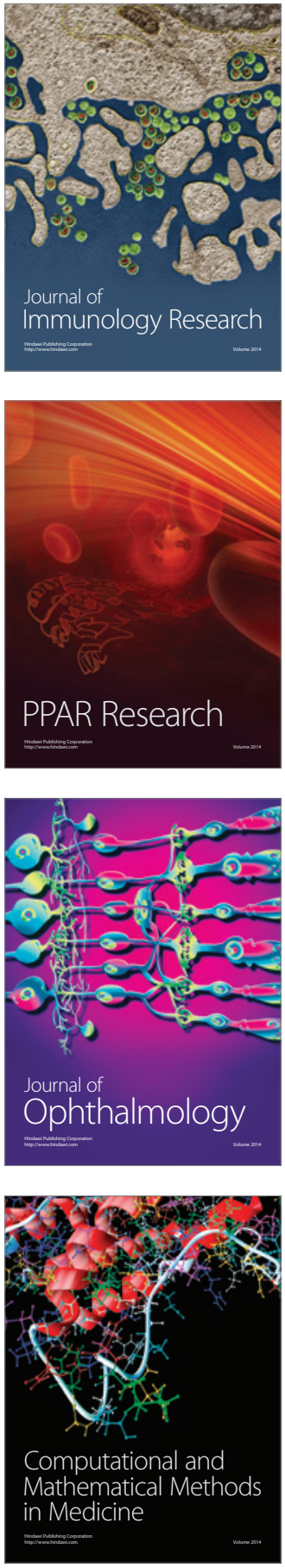

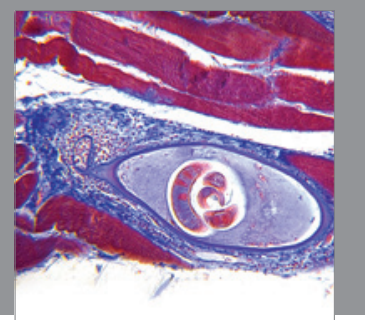

Gastroenterology

Research and Practice
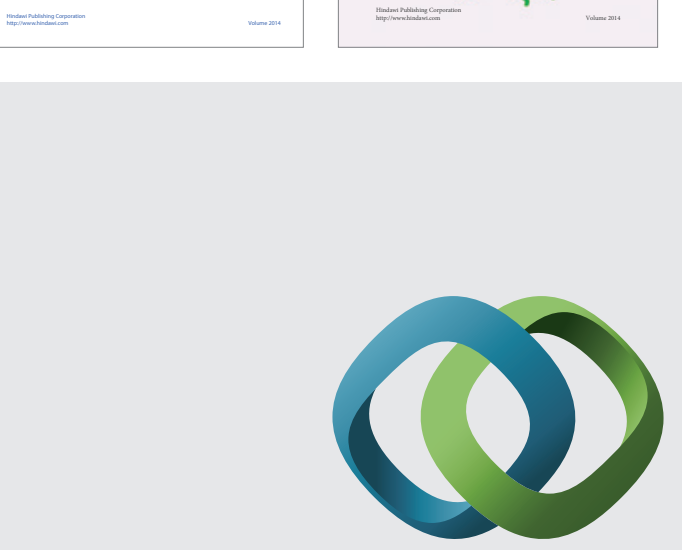

\section{Hindawi}

Submit your manuscripts at

http://www.hindawi.com
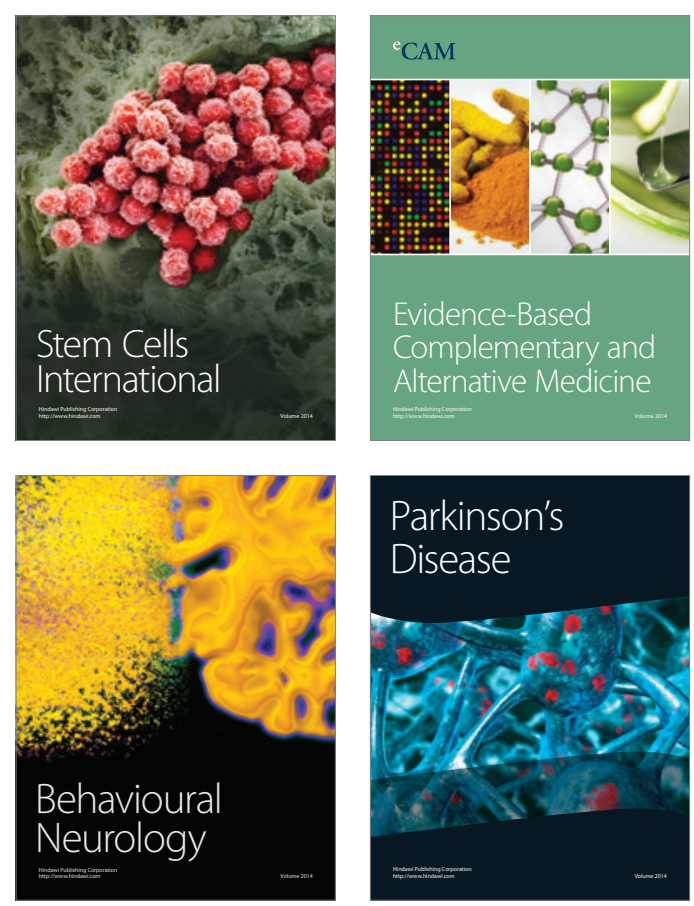

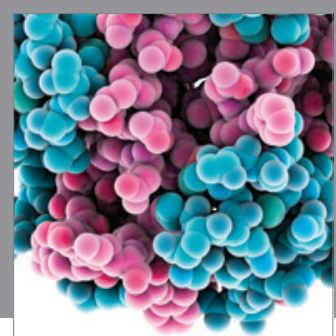

Journal of
Diabetes Research

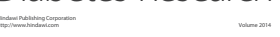

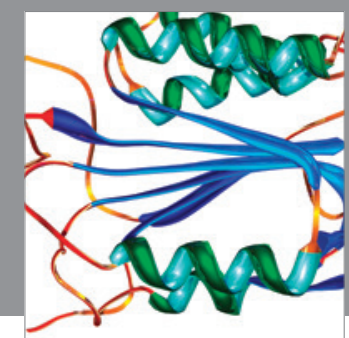

Disease Markers
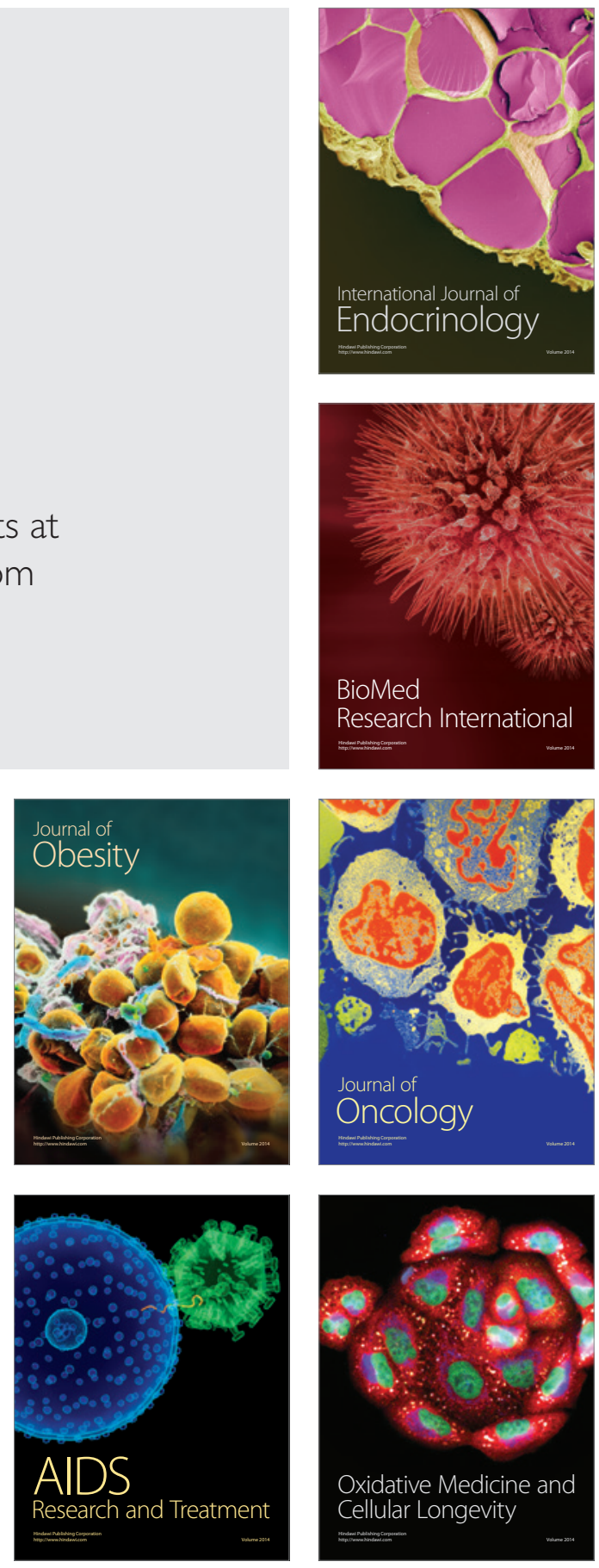